\title{
X-ray Absorption Spectroscopic Studies on Light-Induced Excited Spin State Trapping of an Fe(II) Complex
}

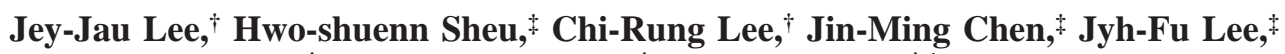 \\ Chih-Chieh Wang, ${ }^{\dagger}$ Chun-Hsun Huang, ${ }^{\dagger}$ and Yu Wang*,,,+
}

\author{
Contribution from the Department of Chemistry, National Taiwan University, Taipei, Taiwan, R.O.C., and \\ the Synchrotron Radiation Research Center, Hsin Chu, Taiwan, R.O.C.
}

Received December 10, 1999

\begin{abstract}
Light-induced excited spin state trapping of $\mathrm{Fe}(\mathrm{phen})_{2}(\mathrm{NCS})_{2}$ is studied by X-ray absorption spectroscopy using synchrotron radiation. The molecular structure of the excited high-spin state at $17 \mathrm{~K}$ is investigated by EXAFS. The relaxation of the light-induced high-spin state at various temperatures is monitored through the absorption spectra. The concurrent abrupt lattice change with the change in electronic configuration is observed through single crystal X-ray diffraction. The lattice cooperative effect is important in the relaxation behavior. The magnetic property and the relaxation behavior of two differently prepared samples are distinctly different.
\end{abstract}

\section{Introduction}

The interesting spin-crossover phenomenon, ${ }^{5} \mathrm{~T}_{2}, S=2$ (high spin, HS) $\leftrightarrow{ }^{1} \mathrm{~A}_{1}, S=0$ (low spin, LS) of the molecular complex, $\mathrm{Fe}^{\mathrm{II}}$ (phen $)_{2}(\mathrm{NCS})_{2}$, has been extensively studied over the past 30 years by various techniques. Magnetic susceptibility clearly showed an abrupt spin transition at $176 \mathrm{~K}^{1,2}$ and the molar heat capacity, $C_{\mathrm{p}}$, yielded a big peak at $176 \mathrm{~K}^{3}$ as well. Electronic spectral analysis in the region characteristic of the $\mathrm{d}-\mathrm{d}$ transition indicated an increase in the ligand field parameter(10 Dq) of $0.55 \mathrm{eV}$ from a high-spin quintet state to a low-spin singlet state transition. ${ }^{2}$ Single-crystal X-ray diffraction measurements at two temperatures $(293,130 \mathrm{~K})^{4,5}$ showed a decrease in the $\mathrm{Fe}-\mathrm{N}$ distance of 0.2 and $0.1 \AA$, respectively, for the $\mathrm{Fe}-\mathrm{N}_{\text {phen }}$ and $\mathrm{Fe}-\mathrm{N}_{\mathrm{NCS}}$ bonds as well as changes in $\angle \mathrm{N}-\mathrm{Fe}-\mathrm{N}$ angles, leading to a more regular octahedral shape at low temperature. Such a contraction of the first coordination shell was also detected in EXAFS analysis. ${ }^{6}$ Furthermore, the spin transition of $\mathrm{Fe}^{\mathrm{II}}$ was carefully monitored by Mössbauer measurements, ${ }^{7}$ and the $\mathrm{X}$ - ray absorption spectra of the iron $\mathrm{K}$ and $\mathrm{L}_{\mathrm{II}}$ III edges of this complex show significant modifications during the spin transition. ${ }^{6,8,9} \mathrm{~A}$ shift of 1.44 and $0.5 \mathrm{eV}$ in $\mathrm{L}_{\mathrm{III}}$ and $\mathrm{L}_{\mathrm{II}}$ absorption edge toward high energies was detected on going from 300 (HS) to $77 \mathrm{~K}$ (LS). ${ }^{8,9}$ Recent crystal field multiplet calculations ${ }^{10}$ could actually well reproduce the experimental spectra.

\footnotetext{
National Taiwan University.

\$Synchrotron Radiation Research Center.

(1) König, E.; Madeja, K. Chem. Commun. 1966, 3, 61.

(2) König, E.; Madeja, K. Inorg. Chem. 1967, 6, 48.

(3) Sorai, M.; Seki, S. J. Phys. Chem. Solids 1974, 35, 555.

(4) Gallois, B.; Real, J.-A.; Hauw, C.; Zarembowitch, J. Inorg. Chem. 1990, 29, 1152 .

(5) Real, J.-A.; Gallois, B.; Granier, T.; Franz, S.-P.; Zarembowitch, J. Inorg. Chem. 1992, 31, 4972.

(6) Cartier, C.; Thuery, P.; Verdaguer, M.; Zarembowitch, J.; Michalowicz, A. J. Phys. C8, 1986, 47, 563.

(7) Goodwin, H. A. Coord. Chem. Rev. 1976, 18, 293

(8) Cartier dit Moulin, C.; Sainctavit, P.; Briois, V. In Proceedings of the Seventh International Conference on X-Ray Absorption Fine Structure, Kobe, Japan, 1992. Jpn. J. Appl. Phys. 1993, 32, 38.

(9) Cartier dit Moulin, C.; Rudolf, P.; Flank, A.-M.; Chen, C. T. J. Phys. Chem. 1992, 96, 6196.
}

Evidence of the light-induced excited spin state trapping (LIESST) $^{11-14}$ in this complex was found in Mössbauer spectroscopy ${ }^{12,13}$ and in FTIR spectroscopy. ${ }^{14}$ The trapping of the metastable HS state was detected at temperatures below 55 $\mathrm{K}$ after the sample was irradiated with a xenon lamp and appropriate filter ${ }^{12}$ or $\mathrm{He} / \mathrm{Ne}$ laser. ${ }^{14}$

The K-edge absorption at the near-edge range (XANES) is known to be sensitive to the coordination symmetry and the oxidation state of the target atom. The preedge peak was recently found ${ }^{15}$ to be rich in information about the electron density distribution on $3 \mathrm{~d}$ orbitals. The distinct change in K-edge absorption spectra of $\mathrm{Fe}(\mathrm{phen})_{2}(\mathrm{NCS})_{2}$ during the spin transition was observed. ${ }^{8,9}$ The L-edge absorption spectroscopy is very sensitive to the electronic changes of the target atom, especially the changes involving the modification on the $3 \mathrm{~d}$ orbital populations such as the spin-crossover phenomenon. Therefore, the soft X-ray absorption spectroscopies (K- and L-edge) are ideal for the study of LIESST in this iron(II) complex. The earlier report of the HS/LS K- and $\mathrm{L}_{\mathrm{II}, \mathrm{III}}$-edge absorption spectra $^{4,8,9}$ above and below the transition temperature gives a solid base for this investigation. This work presents the first example of applying the K-, L-edge absorption technique to examine the LIESST phenomenon. Although the K-edge absorption spectrum, is not as sensitive to the variation of $3 \mathrm{~d}$ orbital population as the L-edge absorption spectrum, it does reflect the changes of the population of the antibonding orbital of $\mathrm{Fe}-\mathrm{N}$ due to the spin transition of the $\mathrm{Fe}^{\mathrm{II}}$ ion.

The mechanism of such discontinuous thermal- and lightinduced spin transitions of $\mathrm{Fe}^{\mathrm{II}}$ complexes has drawn wide attention, especially after the Mössbauer technique was intro-

(10) Briois, V.; Cartier dit Moulin, C.; Sainctavit, P.; Brouder, C.; Flank, A.-M. J. Am. Chem. Soc. 1995, 117, 1019.

(11) Decurtins, S.; Gütlich, P.; Köhler, C. P.; Spiering, H. Chem. Phys. Lett. 1984, 105, 1.

(12) Decurtins, S.; Gütlich, P.; Köhler, C. P.; Spiering, H. J. Chem. Soc., Chem. Commun. 1985, 340.

(13) Decurtins, S.; Gütlich, P.; Hasselbach, K. M.; Hauser, A.; Spiering, H. Inorg. Chem. 1985, 24, 2174.

(14) Herber, R.; Casson, L. M. Inorg. Chem. 1986, 25, 847.

(15) Westre, T.E.; Kennepohl, P.; DeWitt, J. G.; Hedman, B.; Hodgson, K. O.; Solomon, E. I. J. Am. Chem. Soc. 1997, 119, 6297. 
duced. A couple of excellent reviews ${ }^{16,17}$ on this topic give a thorough discussion of all possible models. A pulse laser photolysis study on the relaxation of a polymer-doped highspin $\mathrm{Fe}^{\mathrm{II}}$ complex ${ }^{18}$ indicates the relevance of a quantum mechanical tunneling mechanism at temperatures below $120 \mathrm{~K}$. The LIESST phenomenon in the crystalline state is believed to be associated with the elastic interactions and lattice cooperativity. ${ }^{16-19}$ The lattice defects and lattice anisotropy are also considered ${ }^{19-21}$ for such a phenomenon. The HS-LS relaxation kinetics after laser excitation in a series of iron(II) complexes was carefully studied by Hauser et al., ${ }^{19,22-26}$ who proposed a thermally activated relaxation behavior or a nonadiabatic tunneling at elevated temperatures and a nearly temperature independent quantum mechanical tunneling mechanism at low temperatures $(<100 \mathrm{~K})$. The intersystem crossings between singlets, triplets, and quintets as well as the reverse-LIESST are well illustrated in the case of $\left[\mathrm{Fe}(\mathrm{ptz})_{6}\right]\left(\mathrm{BF}_{4}\right)_{2} \cdot{ }^{17,22,27} \mathrm{~A}$ study on $\left[\mathrm{Fe}_{0.35} \mathrm{Ni}_{0.65}(\mathrm{mtz})_{6}\right]\left(\mathrm{ClO}_{4}\right)_{2}$ shows that there are two transition states $(220,120 \mathrm{~K})$, each giving very different relaxation behavior. ${ }^{28}$ The detailed mechanism for the spin transition and LIESST is still not completely clear and may be worth some more effort; the XAS should be a very useful tool for this purpose.

\section{Experimental Section}

The iron complex is prepared according to the literature. ${ }^{4,29} \mathrm{~L}_{\mathrm{II}, \mathrm{III}}{ }^{-}$ edge absorption spectra are measured on the high-energy spherical grating monochromator (HSGM) beam line of SRRC. The spectra are recorded by the total electron yield mode using a microchannel plate (MCP) detector. The schematic experimental setup of the instrument is illustrated in Figure 1. The photon energy is calibrated using 708.5 $\mathrm{eV}$ for the peak of $\mathrm{L}_{\mathrm{III}}$ line of $\alpha-\mathrm{Fe}_{2} \mathrm{O}_{3}$; the entrance and exit slits are set at $30 \times 30$ um corresponding to an energy resolution of $0.25 \mathrm{eV}$ at $\sim 700 \mathrm{eV}$ range. XANES and EXAFS spectra are measured on the 1.8T/ 25 pole wiggler beam line using fluorescence mode with a $6-\mu \mathrm{m}$ metal mesh made out of $\mathrm{Mn}$. The resolving power of $\Delta E / E$ is up to $1 / 7000$ (operational energy of $1.5 \mathrm{GeV}, 100-200 \mathrm{~mA}$ ). The $\mathrm{Si}(111)$ double crystal is employed as the monochromator. The energy calibration is made on the basis of the $E_{0}$ of iron foil at $7112 \mathrm{eV}$. An APD unit is used to control the temperature; the sample is pressed on a In foil to ensure the good thermal conductivity. The pressure of the measuring chamber is at $\sim 10^{-9}$ and $10^{-3}$ Torr for L-edge and K-edge absorption, respectively.

Light-induced excitation is undertaken by a He/Ne laser or a Xe arc lamp with the proper filter. The excitation normally takes place in a few minutes. The light is then turned off before making the scan of the spectra.

The EXAFS oscillation function $k^{3} \chi(k)$ was obtained by a standard analysis procedure ${ }^{30,31}$ using the UWXAFS code (including

(16) Gütlich, P.; Hauser, A.; Spiering, H. Angew Chem., Int. Ed. Engl. 1994, 33, 2024-2054.

(17) Gütlich, P.; Hauser, A. Coord. Chem. Rev. 1990, 97, 1.

(18) Xie, C.-L.; Hendrickson, D. N. J. Am. Chem. Soc. 1987, 109, 6981

(19) Hauser, A.; Gütlich, P.; Spiering, H. Inorg. Chem. 1986, 25, 4245. 10089

(20) Spiering, H.; Willenbacher, N. J. Phys: Condens. Matter 1989, 1,

(21) Granier, T.; Gallois, B.; Gaultier, J.; Real, J.-A.; Zarembowitch, J. Inorg. Chem. 1993, 32, 5305.

(22) Hauser, A. Coord. Chem. Rev. 1991, 111, 275.

(23) Hauser, A.; Vef, A.; Adler, P. J. Chem. Phys. 1991, 95, 8710.

(24) Hauser, A. J. Chem. Phys. 1991, 94, 2741.

(25) Hauser, A. Chem. Phys. Lett. 1992, 192, 65.

(26) Hauser, A.; Adler, J.; Gütlich, P. Chem. Phys. Lett. 1988, 152, 468.

(27) Hauser, A. Chem. Phys. Lett. 1986, 124, 543.

(28) Buchen, T.; Schollmeyer, D.; Gütlich, P. Inorg. Chem. 1996, 35, 155 .

(29) Schilt, A. A.; Fritsch, K. J. Inorg. Nucl. Chem. 1966, 28, 2677.

(30) Sayers, D. E.; Bunker, B. A. X-Ray Absorption: Principles, Applications, Techniques of EXAFS, SEXAFS and XANES; Koningsberger, D. C., Prins, R., Eds.; John Wiley \& Sons: NewYork, 1988; p 211.

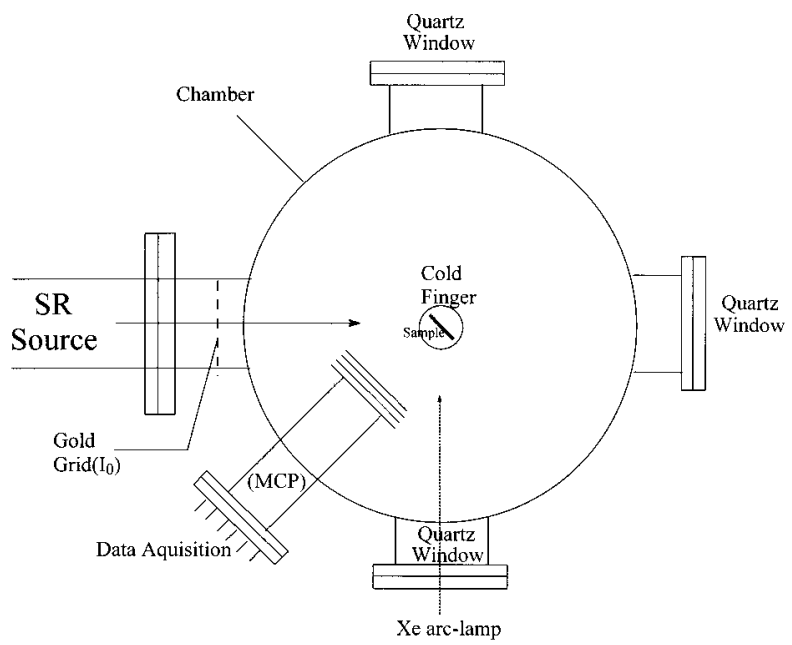

Figure 1. Schematic drawing of the experimental setup for L-edge absorption.

AUTOBK, ${ }^{32-34}$ ATOMS, ${ }^{35}$ FEEE, ${ }^{36,37}$ and FEFFIT ${ }^{32,38}$ programs): preedge baseline and postedge background subtractions are applied by a linear and by a spline function, respectively. The analysis of the first coordination shell of iron $\left(\mathrm{Fe}-\mathrm{N}_{6}\right)$ is regarded as a single scattering path, and only the first shell contribution is considered in this work. The iron atom is at the $C_{2}$ symmetry site, and the starting point of $\mathrm{Fe}-\mathrm{N}$ bond lengths is based on the single-crystal diffraction (XRD) data at room temperature ${ }^{29}$ and at $20 \mathrm{~K}$ for HS-1 and LS-1, respectively.

Single-crystal diffraction data at $20 \mathrm{~K}$ is taken at the X3A1 beam line of NSLS at BNL, using a wavelength of $0.642 \AA$. A total of 7433 reflections are collected on IP. Intensity integration and scale are based on the DENZO and the SCALE programs of the HKL package, ${ }^{39}$ for the data analyses and structure solution the NRCSDP program are used. ${ }^{40}$

FTIR spectra are taken on a Bomem spectrometer using $\mathrm{KBr}$ tablets; the sample temperature is controlled by an APD unit. The magnetic measurement is made on a SQUID magnetometer in the temperature range of $5-300 \mathrm{~K}$.

\section{Results and Discussion}

Thermally Induced Spin Transition. Variation of the magnetic moments, $\mu_{\text {eff }}$, with temperature for the iron compound is shown in Figure 2, which reconfirms the abrupt spin crossover at $176 \mathrm{~K}$. Figure 2 also confirms the previously observed ${ }^{41}$ clear difference between the two samples prepared by high-temperature extraction ${ }^{41,4}$ and by diffusion. ${ }^{29}$ The extraction sample undergoes a complete spin transition from ${ }^{5} \mathrm{~T}_{2}$ to ${ }^{1} \mathrm{~A}_{1}$, while the diffusion sample displays some residual magnetic moment $\left(\sim 1.8 \mu_{\mathrm{B}}\right.$.) at temperatures below $176 \mathrm{~K}$. However, the single crystal can only be obtained by the diffusion method. The spincrossover phenomenon is not accompanied by a crystal structural phase change in this case. Both HS and LS state molecules are crystallized in the same space group, $\mathrm{Pbcn}$; however, the lattice

(31) Asakura, A. X-Ray Absorption Fine Structure for Catalysts and Surface; Iwasawa, Y., Ed.; World Scientific: Singapore, 1996; p 34.

(32) Newville, M. Ph.D. Thesis, 1997.

(33) Newville, M.; Livins, P.; Yacoby, Y.; Rehr, J. J.; Stern, E. A. Phys. Rev. B 1993, 47, 14126.

(34) Newville, M. AUTOBK document, 1995.

(35) Ravel, B. ATOMS document, 1995.

(36) Zabinsky, S. I.; Rehr, J. J.; Ankudinov, A.; Albers, R. C.; Eller, M. J. Phys. Rev. B 1995, 52, 2995.

(37) Ankudinov, A. FEFF601 document, 1995.

(38) Newville, N. FEFFIT document, 1996.

(39) Otwinowsky, Z.; Minor, W. Methods Enzymol. 1997, 276, 307.

(40) Gabe, E. J.; Le Page, Y.; Charland, J.-P.; Lee, F. L.; White, P. S. J. Appl. Crystallogr. 1989, 22, 384.

(41) Ganguli, P.; Gütlich, P.; Müller, E. W. J. Chem. Soc., Dalton Trans. 1981, 441. 


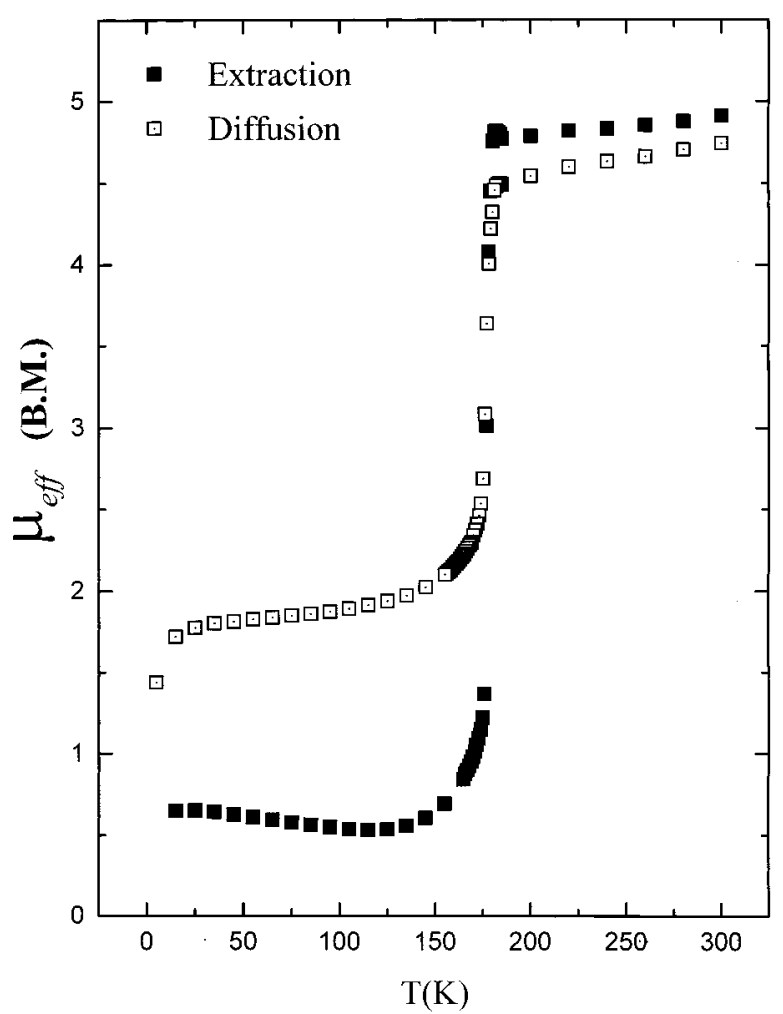

Figure 2. Temperature-dependent effective magnetic moment $\left(\mu_{\mathrm{eff}}\right)$ measurement of $\mathrm{Fe}(\text { phen })_{2}(\mathrm{NCS})_{2}$ prepared by extraction $(\mathbf{\square})$ and diffusion ( $\square$ )methods.

Table 1. Lattice Parameters and $\mathrm{Fe}-\mathrm{N}$ Distances of the HS-1, LS-1, and HS-2 States Based on Single-Crystal Diffraction Data (XRD) and EXAFS Data

\begin{tabular}{|c|c|c|c|c|c|}
\hline $\mathrm{P}$ bcn & \multicolumn{2}{|c|}{ HS-1 (300 K) } & HS-2 & \multicolumn{2}{|c|}{ LS-1 (20 K) } \\
\hline \multicolumn{6}{|c|}{ lattice param $(\AA)$} \\
\hline$a$ & \multicolumn{2}{|c|}{$13.146(2)$} & & \multicolumn{2}{|c|}{$12.510(2)$} \\
\hline$b$ & \multicolumn{2}{|c|}{$10.129(4)$} & & \multicolumn{2}{|c|}{$9.959(3)$} \\
\hline \multirow[t]{2}{*}{$c$} & \multicolumn{2}{|c|}{$17.453(6)$} & & \multicolumn{2}{|c|}{$16.949(5)$} \\
\hline & XRD & EXAFS & EXAFS & XRD & EXAFS \\
\hline$T(K)$ & 300 & 300 & 17 & 20 & 17 \\
\hline $\mathrm{Fe}-\mathrm{N}_{\text {(phen) }}\left(\AA \AA^{\circ}\right)$ & $2.214(8)$ & $2.190(5)$ & $2.12(1)$ & $1.967(3)$ & $1.985(5)$ \\
\hline $\mathrm{Fe}-\mathrm{N}_{\text {(phen) }}(\stackrel{\AA}{\circ})$ & $2.194(8)$ & $2.132(5)$ & $2.06(1)$ & $1.962(2)$ & $1.979(5)$ \\
\hline $\mathrm{Fe}-\mathrm{N}_{(\mathrm{NCS})}(\AA)$ & $2.070(9)$ & $2.115(5)$ & $2.04(1)$ & $1.925(3)$ & $1.943(5)$ \\
\hline
\end{tabular}

contracts anisotropically and the $\mathrm{Fe}-\mathrm{N}$ bond shortens significantly from the HS to the LS state. The results are listed in Table 1. To investigate the change in structure near the transition temperature, a diffraction pattern out of a certain orientation is monitored through an image plate detector. A selected group of reflections out of one frame at various temperatures near 176 $\mathrm{K}$ is carefully studied; the observation of the drastic change in $d$ spacing within a few kelvin implies that even the lattice change (diffraction pattern) during the spin transition is as abrupt as the change in magnetic moment. The lattice parameters determinated in the temperature range of $140-190 \mathrm{~K}$ based on the refinement of 150 reflections out of this frame indicate that the abrupt change in pattern is due mainly to the change in "a" dimension, which can be manifested by the distinct shift in diffraction position of high "h" reflections. The thermally induced spin transitions of both the extraction and the diffusion samples are reconfirmed by FTIR and K- and L- edge X-ray absorption spectra. The results for the extraction samples are shown in Figures 3a, 4a, and 5a, respectively. The ones from the diffusion sample are basically the same as those for the extraction sample, except in the case of the LS state of FTIR,

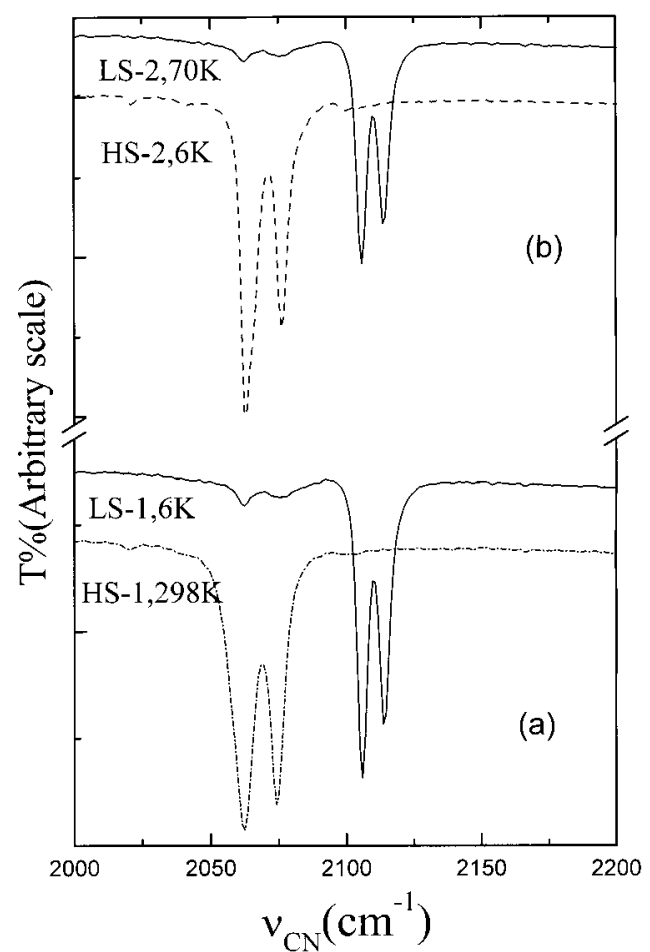

Figure 3. $\mathrm{C}-\mathrm{N}$ streching frequency, $v_{\mathrm{C}-\mathrm{N}}$, of (a) HS-1/LS-1 and (b) HS-2/LS-2 for the extraction sample.

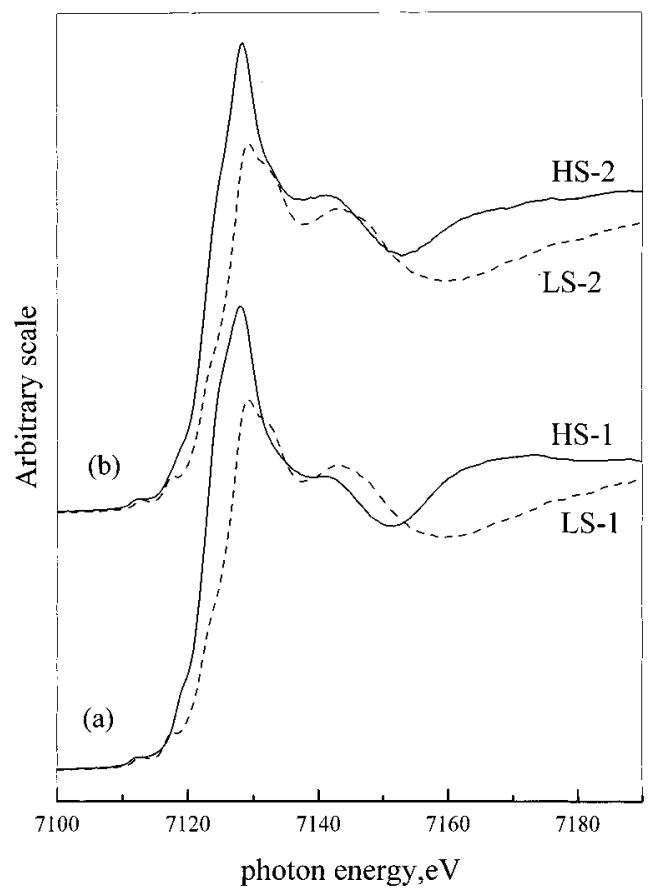

Figure 4. XANES spectra of (a) HS-1/LS-1 and (b) HS-2/LS-2 for the extraction sample.

where a small residual peaks of the HS state is observed. This is consistent with the magnetic measurement.

LIESST and Electronic Configuration of HS-2. LIESST in this $\mathrm{Fe}^{\mathrm{II}}$ complex is investigated by FTIR at $6 \mathrm{~K}$ using a 5 $\mathrm{mW} \mathrm{He} / \mathrm{Ne}$ laser. The sample pressed in a $\mathrm{KBr}$ tablet is exposed to the light source for only a few minutes before complete excitation from the LS to the HS state has taken place. To differentiate this light-induced excited HS state from the thermally induced HS state, we designate the light-induced one HS-2 and the thermally induced one HS-1 after the convention in the literature. ${ }^{14}$ This HS-2 state in the solid is stable for at 


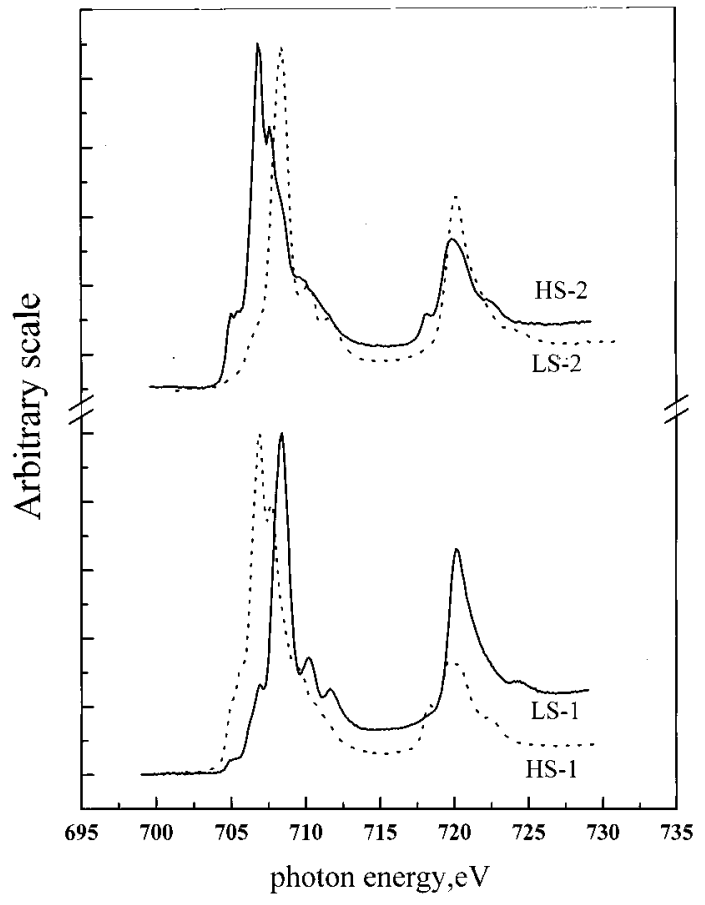

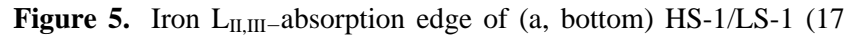
$\mathrm{K})$ and (b, top) HS-2 (17 K)/LS-2 $(70 \mathrm{~K})$ for the extraction sample.

least $10 \mathrm{~h}$ at $17 \mathrm{~K}$ during the measurement. The $\mathrm{C}-\mathrm{N}$ stretching frequency, $v_{\mathrm{C}-\mathrm{N}}$, of HS-2 is shown in Figure $3 \mathrm{~b}$. In the case of iron $\mathrm{K}$ - and L-edge absorption, we have performed the experiment at $17 \mathrm{~K}$ using the Xe arc lamp with a red light filter (650 $\pm 20 \mathrm{~nm}$ ); the exposure time is $\sim 25 \mathrm{~min}$. Again the excitation is complete, trapping of the HS-2 state occurs as expected. The spectra are displayed in Figures $4 \mathrm{~b}$ and $5 \mathrm{~b}$, respectively. A recent report ${ }^{42}$ claimed that the soft $\mathrm{X}$-ray around $700 \mathrm{eV}$ could induce excitation from the LS to the HS state at $\sim 45 \mathrm{~K}$. However, we have not observed such excitation during our experiments, although the white light of the synchrotron radiation does cause some excitation effect and/or damage on the sample when the temperature is $\sim 50 \mathrm{~K}$.

It would be interesting to know the exact electronic configuration and the geometry of the HS-2 state. It is also important to see whether they are the same as those of HS-1. It was mentioned ${ }^{14}$ that there is a blue shift of $4-6 \mathrm{~cm}^{-1}$ in $v_{\mathrm{CN}}$ from HS-1 to HS-2. A small difference in chemical shift $(0.2 \mathrm{~mm}$ $\left.\mathrm{s}^{-1}\right)$ and in quadrupole splitting $\left(0.24 \mathrm{~mm} \mathrm{~s}^{-1}\right)$ was also reported by means of Mössbauer spectra. ${ }^{12}$ The blue shift in $v_{\mathrm{CN}}$ from our observation seems insignificant $\left(\sim 1 \mathrm{~cm}^{-1}\right)$ both for the extraction and for the diffusion sample. However, the difference between the HS-2 and the HS-1 states is detected in XANES and EXAFS spectra. In fact, $\mathrm{Fe}-\mathrm{N}$ distances are determined by EXAFS for HS-1, LS-1, and HS-2; the fitting of the first coordination sphere of $\mathrm{Fe}-\mathrm{N}_{6}$ is displayed in Figure 6. The distances are listed in Table 1 together with the distances obtained from single-crystal X-ray diffraction. ${ }^{29}$ The $\mathrm{Fe}-\mathrm{N}$ distances of HS-1 and LS-1 obtained from EXAFS are not significantly different from those obtained from diffraction data.

According to the EXAFS data, Fe-to-N distances of the HS-2 state are slightly shorter than those of HS-1, but significantly longer than those of the LS-1 state after the temperature effect is taken into account. The difference in the L-edge absorption between HS-2 and HS-1 is mainly due to the temperature effect

(42) Collision, D.; Garner, C. D.; McGrath, C. M.; Mosselmans, J. F. W.; Roper, M. D.; Seddon, J. M. W.; Sinn, E.; Young, N. A. J. Chem. Soc., Dalton Trans., 1997, 4371.

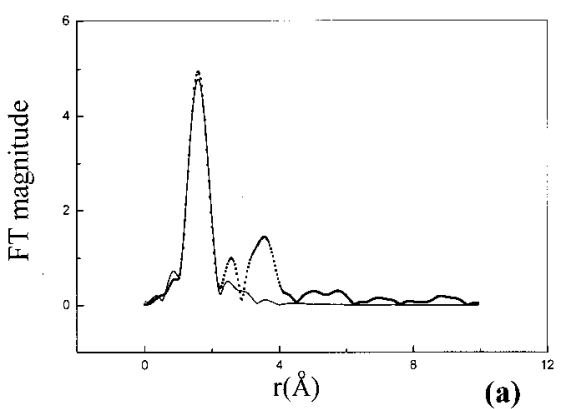

HS-1

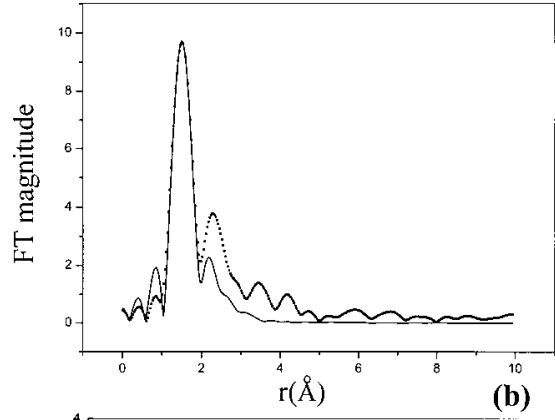

LS-1

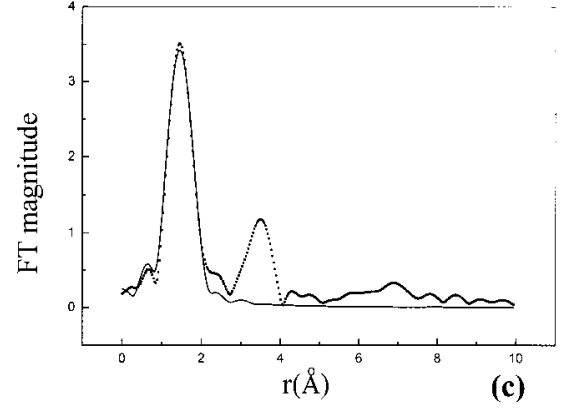

HS-2

Figure 6. Fourier transforms of the experimental EXAFS spectrum $(\cdots)$ and FEFF ab initio model $(-)$ on the first shell of coordination of iron $\left(\mathrm{Fe}-\mathrm{N}_{6}\right)$ for (a) HS-1 (300 K), (b) LS-1 (17 K), and (c) HS-2 (17 K) state.

based on the crystal field multiplet simulation. LS-2 and LS-1 states are basically the same from our observation.

The crystal field multiplet simulation, assuming an $O_{h}$ symmetry at the $\mathrm{Fe}$ atom, gives rise to a $10 \mathrm{Dq}$ value of 0.9 and $2.2 \mathrm{eV}$, respectively, for HS-1 and LS-1; the addition of a metal-to-ligand charge transfer (MLCT) factor is important for fitting of some fine details of peak profile, which was not considered in the earlier report. ${ }^{10}$ The detail comparisons of the measured and calculated curves with and without MLCT are displayed in Figure 7.

On the basis of all the observations, we may conclude that the electronic configuration of HS-2 is the same as that of HS1 ; however, the geometry of HS-2 is slightly different from that of HS-1 with the volume of HS-2 being slightly smaller.

Thermal Relaxation of HS-2. The thermal relaxation from the metastable HS-2 state to the LS-2 state at $57 \mathrm{~K}$ is illustrated in Figure 8, where a series of iron L-edge absorption spectra is monitored at various time periods after the light-induced excitation has taken place. The typical spectra of the HS-1 and LS-1 states are also included to illustrate the change. It clearly depicts the change of spectra from a mainly HS state at $180 \mathrm{~s}$ after the excitation to a typical LS state at $\sim 4000 \mathrm{~s}$.

To clarify the thermally driven conversion of the metastable HS state (HS-2) to LS state (LS-2) process, the fraction of the HS species $\left(\gamma_{\mathrm{HS}}\right)$ is plotted as a function of time (after the excitation light source is turned off) in the temperature range of 49-65 K; the plots are given in Figure 9, based on iron 


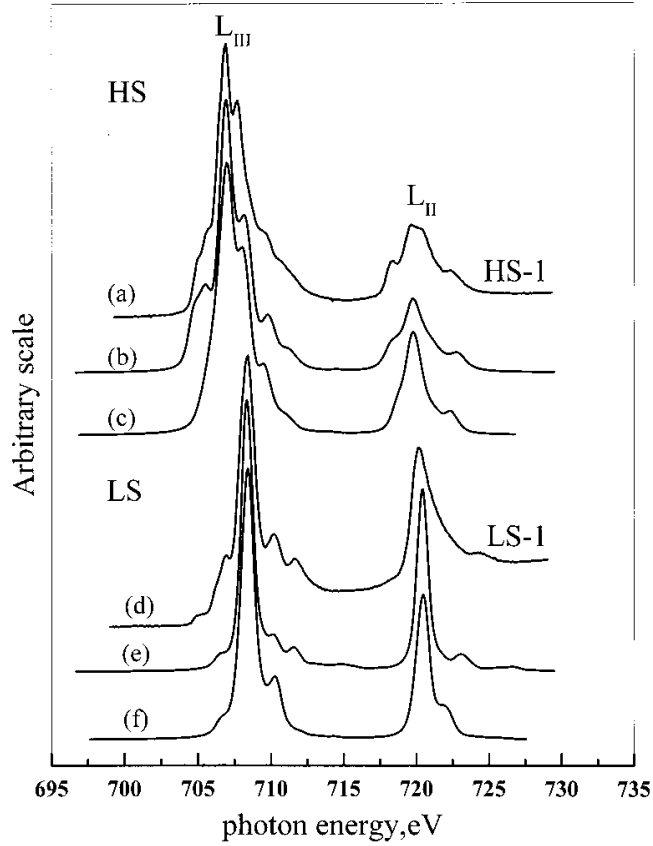

Figure 7. Experimental and calculated $\mathrm{Fe} \mathrm{L}_{\mathrm{II}, \mathrm{III}}$-edge absorption: measured spectra at (a) 298 and (d)17 K; (b, e) calculated spectra including MLCT; (c, f) calculated spectra without MLCT.

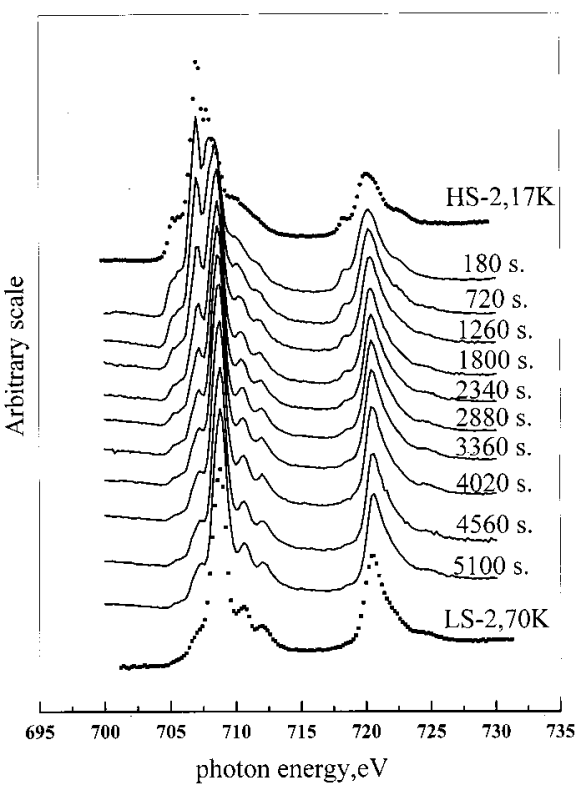

Figure 8. Thermal relaxation of HS-2 at $57 \mathrm{~K}$ based on the L-edge absorption spectra. Typical spectra of HS-2 at $17 \mathrm{~K}$ and of LS-2 at 70 $\mathrm{K}$ are included as dotted curves.

L-edge absorption spectra. There are two diagrams in Figure 9, for the extraction sample (a) and for the diffusion sample (b). It is quite clear that the relaxation rate of the diffusion sample is significantly faster than that of the extraction sample at the same temperature. For example, at $55 \mathrm{~K}$, it takes $\sim 42 \mathrm{~min}$ to be half-relaxed $\left(\gamma_{\mathrm{HS}}=0.5\right)$ for the extraction sample, but only $\sim 20 \mathrm{~min}$ for the diffusion sample. Similar observations are also obtained with K-edge absorption spectra. These observations of the differences in relaxation behavior and in the magnetic measurement from two differently prepared samples is in accord with the theory of elasticity, where the HS or LS molecules are considered to be defects in the crystal lattice. ${ }^{24}$ Thus, the quality of the crystalline material does play a role in the relaxation process. These relaxation curves are sigmoidal, which means

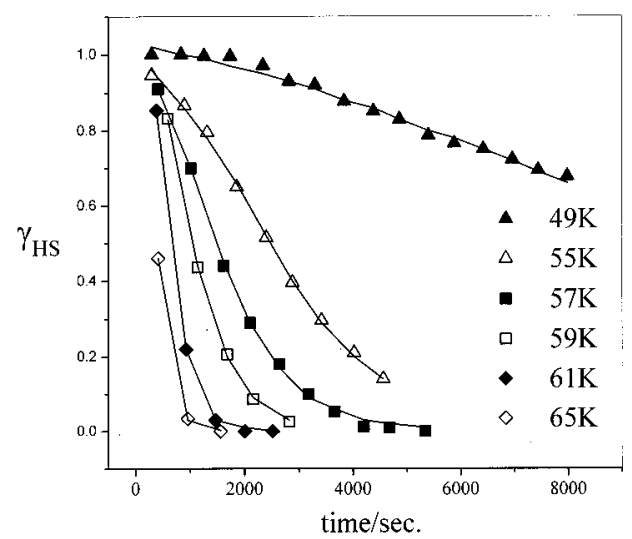

(a)

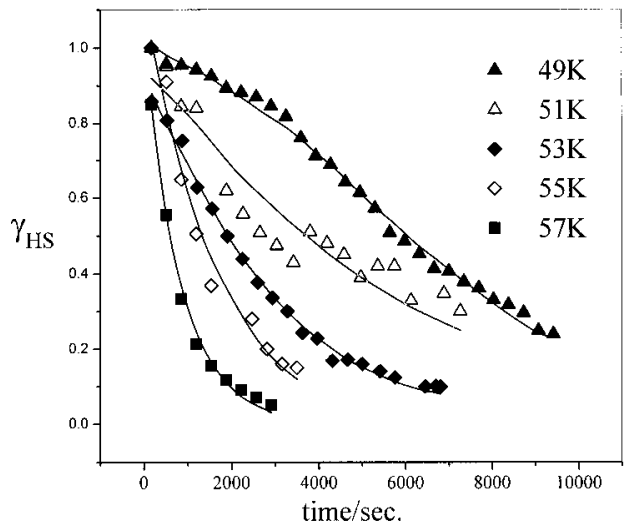

(b)

Figure 9. HS $\rightarrow$ LS relaxation curves at various temperatures based on the L-edge absorption spectra after irradiation on (a) the extraction sample and (b) the diffusion sample. Solid lines are from eq 2.

the relaxation behavior for the complex is not that of an isolated molecule; some cooperative interactions must be included. The model was established for a pure, nondoped $\left[\mathrm{Fe}(\mathrm{ptz})_{6}\right]\left(\mathrm{BF}_{4}\right)_{2}$ spin-crossover complex. ${ }^{25}$ It has been shown that the acceleration in relaxation is due to the buildup of an internal pressure ("lattice pressure") with increasing LS fraction, $\gamma_{\text {LS }}$. Hauser ${ }^{25}$ showed that the relaxation rate is a function of this lattice pressure and thus is a function of the LS fraction, $\gamma_{\text {LS }}$. The relaxation curve can be fitted according to the following equations, ${ }^{22,24-27,43}$

$$
\begin{gathered}
\mathrm{d} \gamma_{\mathrm{LS}} / \mathrm{d} t=k_{\mathrm{HL}}\left(\gamma_{\mathrm{LS}}, T\right)\left(1-\gamma_{\mathrm{LS}}\right) \\
k_{\mathrm{HL}}\left(\gamma_{\mathrm{LS}}, T\right)=k_{\mathrm{HL}}(T) \exp \left[\alpha(T) \gamma_{\mathrm{LS}}\right] \\
k_{\mathrm{HL}}(T)=\mathrm{a}_{\mathrm{HL}} \exp \left(-E_{\mathrm{a}} / k_{\mathrm{B}} T\right)
\end{gathered}
$$

where $k_{\mathrm{HL}}\left(\gamma_{\mathrm{LS}}, T\right)$ is a rate constant for HS $\rightarrow$ LS conversion, which is not only a function of temperature, $T$, but also a function of the LS fraction, $\gamma_{\mathrm{LS}}$. $k_{\mathrm{HL}}\left(\gamma_{\mathrm{LS}}, T\right)$ represents a selfacceleration of HS $\rightarrow$ LS relaxation with increasing $\gamma_{\mathrm{LS}}$ (cooperative effect) as indicated in eq 2, $E_{\mathrm{a}}$ is the activation energy associated with the HS $\rightarrow$ LS relaxation, and $a_{\mathrm{HL}}$ is the preexponential factor. In the temperature range studied, the relaxation behavior can be analyzed using eqs 1 and 2 . The fitting parameters based on the Fe L-edge absorption experiment are summarized in Table 2. The fitted curves are shown in

(43) Létard, J.-F.; Guionneau, P.; Rabardel, L.; Howard, J. A. K.; Goeta, A. E.; Chasseau, D.; Kahn, O. Inorg. Chem. 1998, 37, 4432. 
Table 2. Kinetics Relaxation Model Fitting Parameters from L-Edge Measurements for (a) Extraction and (b) Diffusion Sample

\begin{tabular}{cccrr}
\hline $\mathrm{T}(\mathrm{K})$ & \multicolumn{1}{c}{$k_{\mathrm{HL}}(T)$} & $1 / T$ & $\ln k_{\mathrm{HL}}(T)$ & $\alpha(T)$ \\
\hline \multicolumn{5}{c}{ (a) Extraction Sample } \\
49 & $3.38 \times 10^{-5}$ & 0.02041 & -10.30 & 1.53 \\
55 & $1.47 \times 10^{-4}$ & 0.01818 & -8.83 & 1.27 \\
57 & $2.72 \times 10^{-4}$ & 0.01754 & -8.21 & 1.14 \\
59 & $5.50 \times 10^{-4}$ & 0.01695 & -7.51 & 0.86 \\
61 & $6.00 \times 10^{-4}$ & 0.01639 & -7.42 & 1.40 \\
65 & $9.87 \times 10^{-4}$ & 0.01538 & -6.92 & 1.34 \\
\multicolumn{5}{c}{ (b) Diffusion Sample } \\
49 & $6.16 \times 10^{-5}$ & 0.02041 & -9.69 & 1.26 \\
51 & $1.31 \times 10^{-4}$ & 0.01961 & -8.94 & 0.50 \\
53 & $2.68 \times 10^{-4}$ & 0.01887 & -8.23 & 0.35 \\
55 & $5.65 \times 10^{-4}$ & 0.01818 & -7.48 & 0.15 \\
57 & $1.62 \times 10^{-3}$ & 0.01754 & -6.42 & -0.30 \\
\hline
\end{tabular}

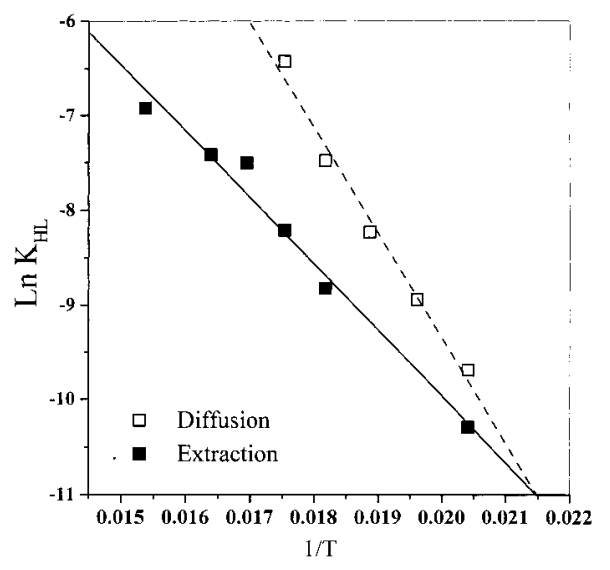

Figure 10. Arrhenius plot of the HS $\rightarrow$ LS relaxation.

Figure 9 as solid lines. Thus, the data of this complex can be understood in terms of a $\gamma_{\mathrm{LS}}$-dependent, self-acceleration process.

According to the theory of nonadiabatic multiphonon relaxation, the temperature independent tunneling rate constant, $k_{H L}^{0}$, is a function of the Huang-Rhys factor $S$ and the reduced energy gap $p . S$ is related to the equilibrium internuclear displacement $\Delta Q$ of the HS and LS states,

$$
S=\frac{1}{2} f \frac{\Delta Q^{2}}{\hbar \omega} \quad \text { where } \quad \Delta Q=\sqrt{6} \Delta \mathrm{r}_{\mathrm{HL}}
$$

$p$ is related to the energy gap, $\Delta \mathrm{E}_{H L}^{0}$, between the lowest vibronic levels of the HS and the LS states according to

$$
p=\Delta \mathrm{E}_{\mathrm{HL}}^{0} / \hbar \omega
$$

where $\omega$ is the frequency of the breathing vibration of the $\mathrm{FeN}_{6}$ core, $\Delta r_{\mathrm{HL}}$ is the difference in $\mathrm{Fe}-\mathrm{N}$ bond length between the HS state and the LS state, and $f$ is the force constant of the $\mathrm{Fe}-\mathrm{N}$ bond.

Two curves of $\ln K_{\mathrm{HL}}$ vs $T^{-1}$ are shown in Figure 10 for the extraction and the diffusion samples, respectively. Both represent linear progression in the temperature range studied $(49-65 \mathrm{~K})$. From these linear plots, one can obtain thermal activation energies, $E_{\mathrm{a}}$, of $487 \pm 34$ and $773 \pm 52 \mathrm{~cm}^{-1}$ and the preexponential factor $a_{\mathrm{HL}}$ of $5.74 \times 10^{1}$ and $3.88 \times 10^{5}$ for the extraction and diffusion samples, respectively. It is obvious that the relaxation rate $K_{\mathrm{HL}}$ is primarily controlled by $a_{\mathrm{HL}}$ in the temperature range studied. Thus $K_{\mathrm{HL}}$ is larger for the diffusion sample than for the extraction sample.

Assuming that, at temperatures below $50 \mathrm{~K}$, the relaxation behavior is temperature independent, then one gets an $S$ value of $\sim 45 \mathrm{~cm}^{-1}$ and $p$ value of $1-2,{ }^{22,24-27}$ Thus, the zero point energy difference of the HS and the LS states, $\Delta \mathrm{E}_{\mathrm{HL}}^{0}$, is $250-$ $500 \mathrm{~cm}^{-1}$. The observed low-temperature tunneling rate, $\mathrm{K}_{\mathrm{HL}}^{0}$ (at $T \rightarrow 0$ ), is $\sim 10^{-10}$, when plotted against the transition temperature $(176 \mathrm{~K})$, which agreees with the literature values for such spin-crossover systems. ${ }^{16,22}$ Unfortunately we cannot afford the lengthy beam time required for study of the relaxation behavior at very low temperature $(<49 \mathrm{~K})$. Neverthless, comparing our work with the relaxation behavior of other spincrossover systems, ${ }^{16,22}$ we believe that the relaxation mechanism does have a temperature-dependent part at somewhat elevated temperatures and a temperature-independent tunneling part at temperatures below $50 \mathrm{~K}$.

\section{Conclusion}

Metal K- and L-edge absorption spectroscopy appears to be a very powerful technique to investigate the spin-crossover system. The abrupt change in diffraction pattern near the transition temperature is firmly observed. LIESST and the relaxation behavior are successfully monitored by X-ray absorption spectroscopy. The model of a self-acceleration of HS to LS relaxation with increasing fraction of LS species is consistent with the experimental observations. The molecular structure of the excited high-spin state (HS-2) at low temperature is believed to be slightly different from that of the normal high-spin state (HS-1). Two differently prepared samples give the same FTIR and XAS spectra, but show significantly different behavior in magnetic measurement and the thermal relaxation behavior of the HS-2 state.

Acknowledgment. We give special thanks to Prof. Y. P. Lee and Miss L. H. Lai of National Tsing-Hwa University for their assistance with the VTFTIR measurement. Thanks are due to Dr. de Groot for supplying the software on crystal field multiplet calculation and to Dr. Otwinowsky, for supplying and kindly helping with the HKL packages. We also o express our thanks to the instrumentation center at National Taiwan University for magnetic measurement and the X3A1 beam line staff for their assistance with the XRD measurement. Financial support from NSC and SRRC are highly appreciated.

Supporting Information Available: HS $\rightarrow$ LS relaxation curves at various temperatures based on the Fe K-edge absorption spectra after irradiation on extraction sample and diffusion sample; tables of crystal data, structure solution and refinement, atomic coordinates, and anisotropic thermal parameters for $\mathrm{Fe}$ $(\text { phen })_{2}(\mathrm{NCS})_{2}$ at $20 \mathrm{~K}$; an Ortep drawing of $\mathrm{Fe}(\text { phen })_{2}(\mathrm{NCS})_{2}$ at $20 \mathrm{~K}$; and Fourier-filtered EXAFS data for the first coordinated shell for HS-1, LS-1, andHS-2. See any current masthead page for ordering information and Web access instructions.

JA9943290 